\title{
Risk Factors and Causes of Short-Term Mortality after Emergency Department Discharge in Older Patients: Using Nationwide Health Insurance Claims Data
}

\author{
Seunggu Na, Yongil Cho, Tae Ho Lim, Hyunggoo Kang, Jaehoon Oh, Byuk Sung Ko \\ Department of Emergency Medicine, Hanyang University College of Medicine, Seoul, Korea
}

Corresponding Author:

Yongil Cho, $\mathrm{MD}, \mathrm{PhD}$

Department of Emergency Medicine,

Hanyang University College of

Medicine, 222-1 Wangsimni-ro,

Seongdong-gu, Seoul 04763, Korea

E-mail: joeguy@hanmail.net

ORCID:

https://orcid.org/0000-0001-5027-6345

Received: July 19, 2019

Revised: September 10, 2019

Accepted: September 16, 2019

\begin{abstract}
Background: The purpose of this study was to identify the risk factors and causes of short-term mortality after emergency department (ED) discharge in older patients. Methods: This population-based cohort study used nationwide health insurance claims data in Korea from 2008 to 2014. The causes of death and diagnoses of patients who died within 1 week after discharge from EDs (1-week ED death) were obtained. The risk factors for 1-week ED death were calculated using Cox proportional hazard regression analyses. Results: The rate of 1-week ED death was $0.5 \%$ among 133,251 individuals aged $\geq 65$ years discharged from EDs. In multivariate analysis, the top five ED discharge diagnoses associated with an increased risk of 1-week ED death were hypotension and vascular disease (adjusted hazard ratio $[\mathrm{aHR}]=5.11 ; 95 \%$ confidence interval [Cl], 3.03-8.63), neoplasm (aHR=4.89; 95\% Cl, 3.77-6.35), coronary artery disease (aHR=3.83; $95 \% \mathrm{Cl}, 2.73-5.39)$, dyspnea $(\mathrm{aHR}=3.41 ; 95 \% \mathrm{Cl}, 2.48-4.68)$, and respiratory disease $(\mathrm{aHR}=2.25$; $95 \% \mathrm{Cl}, 1.73-2.92)$. The most common causes of 1-week ED death were neoplasm (14.8\%), senility (13.8\%), and cerebrovascular disease (11.7\%). Conclusion: Neoplasm, coronary artery disease, and respiratory disease were the discharge diagnoses associated with an increased risk of short-term mortality after ED discharge. Neoplasm was the leading cause of short-term mortality after ED discharge in older patients.
\end{abstract}

Key Words: Aged, Mortality, Emergency departments, Risk factors

\section{INTRODUCTION}

The number of older patients visiting the emergency department (ED) is increasing rapidly. ${ }^{1,2)}$ In the United States, approximately $20 \%$ of the population visits the ED each year, with patients aged 65 years or older accounting for over $45 \%$ of visits. ${ }^{3)}$ According to data from the National Emergency Department Information System in Korea, the proportion of older adults aged 60 years or more increased from $22 \%$ in 2014 to $28 \%$ in 2018 .) Older patients visiting the ED have higher rates of hospitalization, mortality, and repeat visits than those in younger patients. ${ }^{5-7)}$ This is because older patients are more likely to have an ambiguous presentation with multiple comorbidities, as well as complex problems such as polypharmacy and frailty. ${ }^{5-8)}$ Previous studies have reported on early death after discharge from the ED in older adults, with mortality rates of $0.4 \%$ within 30 days and $4.1 \%$ within 90 days. ${ }^{9,10)}$

Several studies have identified common causes of death after discharge from the ED, including neoplasm, ischemic heart disease, cerebrovascular disease, and respiratory disease. ${ }^{11,12)}$ In addition, studies confirming the diagnosis of ED discharge as a risk factor for early death within 7 days after discharge have been reported. $^{13,14)}$ In a study using US Medicare claims data, patients discharged from the ED with altered mentality, dyspnea, or fatigue had higher risks of death within 7 days. ${ }^{13)}$ In another study analyzing the administrative data of 12 EDs in California, high mortality rates were observed within 7 days of ED discharge for diagnoses of noninfectious lung disease, renal disease, and ischemic heart disease. ${ }^{14)}$ However, these studies were performed in adults and the 
causes and risk factors of early death after ED discharge in older populations are not well known.

The purpose of this study was to identify the causes of death in older patients who died after ED discharge and to determine the risk of early death according to the ED discharge diagnoses.

\section{MATERIALS AND METHODS}

\section{Data and Setting}

The present study used data from the National Health Insurance Service (NHIS) senior cohort database. The senior cohort database includes approximately 550,000 randomly sampled comprising $10 \%$ of the 5.5 million older adults aged $\geq 60$ years nationwide. This database includes information on age, sex, income-based health insurance premiums, disease classification codes, treatment, prescription history, and date and cause of death. The causes of death in Statistics Korea, based on death medical certificates issued by physicians, are linked to the NHIS cohort. This study was approved by the Institutional Review Board of Hanyang University Hospital, which waived the requirement for informed consent (No. HYUH 2019-01-029).

\section{Study Population}

We included cases in which older patients aged 65 years or older were discharged from the ED between January 2008 and December 2014. We defined patients who visited EDs using codes from the NHIS in Korea for the management of emergency care; namely, AC101 (regional emergency medical center), AC103 (local emergency medical center), and AC105 (local emergency medical facility). If a patient visited the ED several times, each visit was considered a unique visit. Patients who visited the ED or were hospitalized during the prior 30 days were excluded. We also excluded patients who were admitted or who died on the same day as the ED visit. Finally, patients with duplicate claims were excluded.

\section{Definitions of Variables}

The outcome variable of this study was death within 1 week of the ED visit (1-week ED death). The causes of death were classified according to the International Classification of Disease, 10th Revision (ICD-10) codes (Supplementary Table S1). We investigated characteristics such as age, sex, quintile of the health insurance premiums, and comorbidities. In Korea, health insurance premiums reflect household income levels, in which individuals with higher incomes also have higher health insurance premiums. The comorbidities were defined as cases with one or more diagnostic codes in the inpatient setting or with two or more codes in the outpatient setting in the 3 years before the ED visit
(Supplementary Table S2). ED discharge diagnoses were categorized using ICD-10 codes (Supplementary Table S3). We used only the main diagnostic codes as the ED discharge diagnoses.

\section{Data Analysis}

Among the baseline patient characteristics, continuous variables were presented as median (interquartile range $[\mathrm{IQR}]$ ) categorical variables as frequencies (\%). These baseline characteristics were compared by Wilcoxon rank-sum or Fisher exact tests, as appropriate. The primary outcome was the risk factors for 1-week ED death. Fisher exact tests were performed to determine the difference in the rate of ED discharge diagnosis between the 1-week ED death and survival groups. All significant variables with p-value less than 0.05 in the univariate analysis were included in the multivariate analysis to identify the risk factors for 1-week ED death. Multivariate analysis was performed using Cox proportional hazard regression. Adjusted hazard ratios (aHRs) and confidence intervals (CIs) were

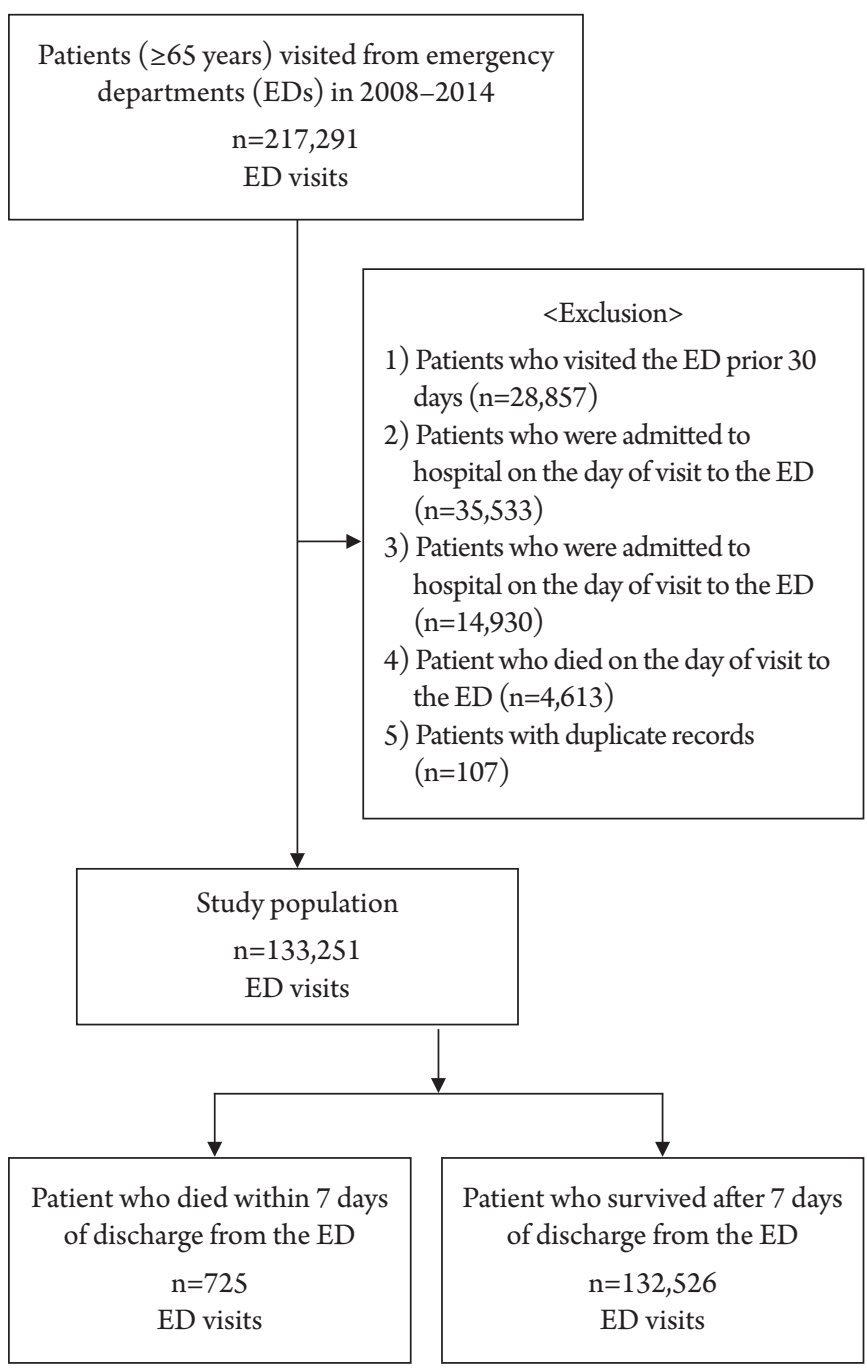

Fig. 1. Study flow diagram. 
calculated by adjusting for age, sex, and income quantiles. Subgroup analysis was performed after dividing the patients into three groups according to the ED level (regional emergency medical center, local emergency medical center, or local emergency medical facility). We identified the discharge diagnoses associated with significantly increased risks of 1-week ED death in the same way as the main analysis. The secondary outcome was the causes of 1-week ED death. Statistical analyses were performed using SAS Enterprise Guide version 7.13 (SAS Institute Inc., Cary, NC, USA) and R software (https://www.r-project.org/).

\section{RESULTS}

\section{Characteristics of the Study Population}

A total of 217,291 cases of older patients aged 65 years or more were discharged from EDs between 2008 and 2014. After excluding $84,040 \mathrm{ED}$ visits, $133,251 \mathrm{ED}$ visits were finally included in the analyses (Fig. 1). Of the included patients, 725 (0.54\%) died with- in 1 week. The basic characteristics of the study population are shown in Table 1 . The median age of the patients was 75 years (IQR, 72-80 years). The median age of the patients in the 1-week ED death group was 81 years (IQR, 75-87 years). The ratio of men was higher in the 1-week ED death group (47\%) than that in the survival group (42.3\%). The 1-week ED death group had higher ratios of comorbidities such as acute myocardial infarction, cancers, arrhythmia, congestive heart failure, renal failure, stroke, and cirrhosis than those in the survival group.

\section{Risk Factors and Causes of 1-week ED death}

Table 2 shows the frequencies and percentages of discharge diagnoses of the ED. Injury and poisoning (14.3\%) was the most common ED discharge diagnosis in the 1-week ED death group, followed by neoplasm (10.6\%) and respiratory disease (10.5\%).

In the multivariate analysis, the risk of 1-week ED death increased by $11 \%(\mathrm{aHR}=1.11 ; 95 \% \mathrm{CI}, 1.10-1.12)$ with age (Table 3$)$. Women had a lower risk of 1-week ED death compared to that in

Table 1. Baseline characteristics of the study population

\begin{tabular}{|c|c|c|c|c|}
\hline & Total $(n=133,251)$ & Survival $(\mathrm{n}=132,526)$ & Death within 1 week $(\mathrm{n}=725)$ & p-value ${ }^{*}$ \\
\hline Age (y) & $75(72-80)$ & $75(72-80)$ & $81(75-87)$ & $<0.001$ \\
\hline \multicolumn{5}{|l|}{ Sex } \\
\hline Male & $56,345(42.3)$ & $56,004(42.3)$ & $341(47.0)$ & 0.010 \\
\hline Female & $76,906(57.7)$ & $76,522(57.7)$ & $384(53.0)$ & \\
\hline Insurance premiums (quintile) & & & & $<0.001$ \\
\hline 1 (lowest income) & $32,809(24.6)$ & $32,598(24.6)$ & $211(29.1)$ & \\
\hline 2 & $12,192(9.1)$ & $12,113(9.1)$ & $79(10.9)$ & \\
\hline 3 & $15,530(11.7)$ & $15,436(11.6)$ & $94(13.0)$ & \\
\hline 4 & $21,993(16.5)$ & $21,879(16.5)$ & $114(15.7)$ & \\
\hline 5 (highest income) & $50,727(38.1)$ & $50,500(38.1)$ & $227(31.3)$ & \\
\hline \multicolumn{5}{|l|}{ Comorbidities (prior 3 years) } \\
\hline Acute myocardial infarction & $6,785(5.1)$ & $6,741(5.1)$ & $44(6.1)$ & 0.240 \\
\hline Rheumatoid arthritis & $15,226(11.4)$ & $15,203(11.5)$ & $63(8.7)$ & 0.019 \\
\hline Asthma & $43,897(33.0)$ & $43,710(33.0)$ & $187(25.8)$ & $<0.001$ \\
\hline All cancers & $21,638(16.2)$ & $21,490(16.2)$ & $148(20.4)$ & 0.003 \\
\hline Arrhythmia & $8,011(6.0)$ & $7,958(6.0)$ & $53(7.3)$ & 0.140 \\
\hline Congestive heart failure & $14,559(10.9)$ & $14,451(10.9)$ & $108(14.9)$ & $<0.001$ \\
\hline Chronic obstructive pulmonary disease & $34,178(25.6)$ & $34,016(25.7)$ & $162(22.3)$ & 0.041 \\
\hline Coronary artery disease (excluding AMI) & $41,563(31.2)$ & $41,366(31.2)$ & $197(27.2)$ & 0.020 \\
\hline Hypertension & $95,173(71.4)$ & $94,691(71.5)$ & $482(66.5)$ & 0.004 \\
\hline Diabetes mellitus & $62,749(47.1)$ & $62,463(47.1)$ & $286(39.4)$ & $<0.001$ \\
\hline Osteoporosis & $49,190(36.9)$ & $49,039(37.0)$ & $151(20.8)$ & $<0.001$ \\
\hline Renal failure & $6,625(5.0)$ & $6,584(5.0)$ & $41(5.7)$ & 0.390 \\
\hline Stroke & $30,939(23.2)$ & $30,750(23.2)$ & $189(26.1)$ & 0.071 \\
\hline Liver cirrhosis & $2,793(2.1)$ & $2,773(2.1)$ & $20(2.8)$ & $<0.001$ \\
\hline
\end{tabular}

Values are presented as median (range) or number (\%).

AMI, acute myocardial infarction.

*Wilcoxon rank-sum test or the Fisher exact test, as appropriate. 
Table 2. Univariate analysis of discharge diagnosis for 1 -weak death after emergency department discharge $(\mathrm{n}=133,251)$

\begin{tabular}{|c|c|c|c|}
\hline & Survival $(\mathrm{n}=132,526)$ & Death within 1 week $(\mathrm{n}=725)$ & p-value* \\
\hline Infectious disease & $7,361(5.6)$ & $28(3.9)$ & 0.050 \\
\hline Neoplasm & $2,943(2.2)$ & $77(10.6)$ & $<0.001$ \\
\hline Hematologic disease & $212(0.2)$ & $2(0.3)$ & 0.325 \\
\hline Endocrine and metabolic disease & $4,460(3.4)$ & $39(5.4)$ & 0.005 \\
\hline Mental and behavioral disease & $1,543(1.1)$ & $6(0.8)$ & 0.718 \\
\hline Nervous system disease & $3,326(2.5)$ & $15(2.1)$ & 0.550 \\
\hline Eye disease & $525(0.4)$ & $0(0.0)$ & 0.126 \\
\hline Ear and mastoid disease & $4,474(3.4)$ & $1(0.1)$ & $<0.001$ \\
\hline Hypertension & $1,166(0.9)$ & $3(0.4)$ & 0.230 \\
\hline Coronary artery disease & $2,064(1.6)$ & $39(5.4)$ & $<0.001$ \\
\hline Non-atherosclerotic heart disease & $1,055(0.8)$ & $7(1.0)$ & 0.528 \\
\hline Cerebrovascular disease & $3,815(2.9)$ & $44(6.1)$ & $<0.001$ \\
\hline Congestive heart failure & $359(0.3)$ & $6(0.8)$ & 0.016 \\
\hline Hypotension and vascular disease & $516(0.4)$ & $15(2.1)$ & $<0.001$ \\
\hline Respiratory disease & $5,701(4.3)$ & $76(10.5)$ & $<0.001$ \\
\hline Hepatobiliary and pancreatic disease & $1,042(0.8)$ & $9(1.2)$ & 0.198 \\
\hline Gastrointestinal disease & $8,874(6.7)$ & $27(3.7)$ & $<0.001$ \\
\hline Skin disease & $2,300(1.7)$ & $5(0.7)$ & 0.030 \\
\hline Non-traumatic musculoskeletal disease & $3,208(2.4)$ & $5(0.7)$ & $<0.001$ \\
\hline Genitourinary disease & $5,887(4.4)$ & $33(4.6)$ & 0.856 \\
\hline Symptom: dizziness & $6,394(4.8)$ & $5(0.7)$ & $<0.001$ \\
\hline Symptom: chest pain & $2627(2.0)$ & $9(1.2)$ & 0.180 \\
\hline Symptom: abdominal pain & $6,163(4.7)$ & $15(2.1)$ & $<0.001$ \\
\hline Symptom: fever & $1,383(1.0)$ & $9(1.2)$ & 0.579 \\
\hline Symptom: dyspnea & $2,201(1.7)$ & $46(6.3)$ & $<0.001$ \\
\hline Symptom: other symptom & $12,089(9.1)$ & $70(9.7)$ & 0.605 \\
\hline Injury and poisoning & $37,568(28.3)$ & $104(14.3)$ & $<0.001$ \\
\hline Other disease & $329(0.2)$ & $4(0.6)$ & 0.110 \\
\hline
\end{tabular}

Values are presented as number (\%).

${ }^{*}$ Fisher exact tests.

men $(\mathrm{aHR}=0.77 ; 95 \% \mathrm{CI}, 0.66-0.89)$. Patients with the fifth quintile of insurance premiums (the highest income group) had a $27 \%$ $(\mathrm{aHR}=0.73$; 95\% CI, 0.60-0.88) lower risk of 1-week ED death than that in patients with incomes in the first quintile. The ED discharge diagnosis with the highest risk of 1-week ED death was hypotension and vascular disease $(\mathrm{aHR}=5.11$; 95\% CI, 3.03-8.63). The most common disease in this group was hypotension $(n=6$, $40 \%)$. The diagnoses at ED discharge with the second-highest risk of 1-week ED death were neoplasm ( $\mathrm{aHR}=4.89$; 95\% CI, 3.776.35), coronary artery disease (CAD) $(\mathrm{aHR}=3.83$; 95\% CI, $2.73-$ 5.39), symptom of dyspnea ( $\mathrm{aHR}=3.41$; 95\% CI, 2.48-4.68), and respiratory disease $(\mathrm{aHR}=2.25 ; 95 \% \mathrm{CI}, 1.73-2.92)$. In addition, patients discharged with cerebrovascular disease and endocrine metabolic disease had an increased risk of 1-week ED death.

The diagnosis with the lowest risk of mortality was ear and mastoid disease $(\mathrm{aHR}=0.05 ; 95 \% \mathrm{CI}, 0.01-0.36)$. In addition, the risks of 1-week ED death were lower in patients with discharge diagnoses of dizziness, non-traumatic musculoskeletal disease, abdominal pain, injury and poisoning, and gastrointestinal disease.

The causes of death for 1-week ED death are shown in Table 4. Neoplasm (14.8\%) was the most frequent cause of death, followed by senility $(13.8 \%)$, cerebrovascular disease $(11.7 \%)$, injury and poisoning $(11.4 \%)$, and $\mathrm{CAD}(9.8 \%)$.

\section{Subgroup Analysis}

In subgroup analysis, neoplasm, $\mathrm{CAD}$, and symptom of dyspnea had increased risks, the same as observed in the main analysis of total included patients of total included patients (Table 5). Local emergency medical facilities had the highest aHR of hypotension and vascular disease $(\mathrm{aHR}=9.17 ; 95 \% \mathrm{CI}, 4.03-20.91)$, followed by neoplasm $(\mathrm{aHR}=6.49 ; 95 \% \mathrm{CI}, 3.91-10.76)$, CAD $(\mathrm{aHR}=5.59 ; 95 \% \mathrm{CI}, 3.14-9.96)$, and symptom of dyspnea 
Table 3. Multivariate analysis investigating risk factors for 1 -weak death after emergency department discharge $(\mathrm{n}=133,251)$

\begin{tabular}{|c|c|c|c|}
\hline & Adjusted HR* & $95 \% \mathrm{CI}^{*}$ & p-value ${ }^{*}$ \\
\hline Age & 1.11 & $1.10-1.12$ & $<0.001$ \\
\hline Sex, female & 0.77 & $0.66-0.89$ & $<0.001$ \\
\hline \multicolumn{4}{|l|}{ Insurance premiums (quintile) } \\
\hline 2 & 1.12 & $0.86-1.45$ & 0.400 \\
\hline 3 & 1.01 & $0.79-1.29$ & 0.921 \\
\hline 4 & 0.9 & $0.71-1.13$ & 0.357 \\
\hline 5 (highest income) & 0.73 & $0.60-0.88$ & $<0.001$ \\
\hline \multicolumn{4}{|c|}{ Discharge diagnosis in the emergency department } \\
\hline Hypotension and vascular disease & 5.11 & $3.03-8.63$ & $<0.001$ \\
\hline Neoplasm & 4.89 & $3.77-6.35$ & $<0.001$ \\
\hline Coronary artery disease & 3.83 & $2.73-5.39$ & $<0.001$ \\
\hline Symptom: dyspnea & 3.41 & $2.48-4.68$ & $<0.001$ \\
\hline Respiratory disease & 2.25 & $1.73-2.92$ & $<0.001$ \\
\hline Congestive heart failure & 2.07 & $0.92-4.68$ & 0.079 \\
\hline Cerebrovascular disease & 2.02 & $1.46-2.79$ & $<0.001$ \\
\hline Endocrine and metabolic disease & 1.59 & $1.13-2.24$ & 0.007 \\
\hline Gastrointestinal disease & 0.58 & $0.39-0.86$ & 0.007 \\
\hline Injury and poisoning & 0.51 & $0.41-0.65$ & $<0.001$ \\
\hline Skin disease & 0.47 & $0.19-1.13$ & 0.093 \\
\hline Symptom: abdominal pain & 0.46 & $0.27-0.78$ & 0.004 \\
\hline Non-traumatic musculoskeletal disease & 0.29 & $0.12-0.71$ & 0.007 \\
\hline Symptom: dizziness & 0.16 & $0.07-0.39$ & $<0.001$ \\
\hline Ear and mastoid disease & 0.05 & $0.01-0.36$ & 0.003 \\
\hline
\end{tabular}

$\mathrm{HR}$, hazard ratio; CI, confidence interval.

${ }^{*}$ Cox proportional hazard regression analyses.

$(\mathrm{aHR}=4.79 ; 95 \% \mathrm{CI}, 2.97-7.74)$.

\section{DISCUSSION}

In this study, the rate of 1 -week ED death was $0.5 \%$. The top five discharge diagnoses associated with an increased risk of short-term mortality were hypotension and vascular disease, neoplasm, CAD, symptom of dyspnea, and respiratory disease. The most common causes of death were neoplasm (14.8\%), senility (13.8\%), cerebrovascular disease (11.7\%), injury and poisoning (11.4\%), and CAD (9.8\%).

Gabayan et al reported a rate of 1-week ED death of $0.05 \%$ among 475,829 patients from 12 hospitals in California discharged from the ED. ${ }^{14)}$ Obermeyer et al. ${ }^{13)}$ studied $10,093,678$ patients included in a $20 \%$ sample of the US Medicare population, reporting a 1 -week mortality rate of $0.12 \%$. The mean ages of the subjects included in these two studies were 47 and 62 years, respectively. Our study of older patients with a median age of 75 showed a 1-weak ED mortality rate of $0.5 \%$.

Multivariate analysis of the association between ED discharge diagnosis and 1-weak ED death showed the highest risk for hypo- tension and vascular disease $(\mathrm{aHR}=5.11)$. These results were not reported in previous studies and indicate that older patients with hypotension or vascular disease should be more seriously considered for hospitalization. In addition, as hypotension itself may be a secondary change rather than a primary diagnosis, additional evaluation should be considered. Neoplasm had an increased risk $(\mathrm{aHR}=4.89)$, consistent with previous studies. Gabayan et al. $\left.{ }^{14}\right)$ reported an odds ratio (OR) of 3.7 for 1-week ED death due to neoplasm in patients with a mean age of 47 years. The median age of the patients in our study was 75 years and the aHR for shortterm ED death in patients with neoplasm was 4.89. Rivera et al. ${ }^{15)}$ analyzed cancer patients who visited the ED, reporting that complications such as pneumonia, septicemia, heart failure, and ileus were associated with hospitalization. Therefore, hospitalization should be considered for patients with neoplasms even if they have the same disease. The risk of 1 -week ED death in $\mathrm{CAD}$ patients increased by 3.83 -fold, similar to previous studies. ${ }^{13,16)}$ Gabayan et al. ${ }^{14)}$ reported an $\mathrm{OR}$ of $\mathrm{CAD}$ of 3.8. More careful observation is needed for patients discharged from the $\mathrm{ED}$ with $\mathrm{CAD}$ diagnosis or related symptom such as chest pain. In the present study, increased risks were observed for patients with dyspnea 
Table 4. Causes of death in patients who died within 1 week of discharge from the emergency department $(n=725)$

\begin{tabular}{lc}
\hline Cause of death & Frequency $(\%)$ \\
\hline Neoplasm & $107(14.8)$ \\
Senility & $100(13.8)$ \\
Cerebrovascular disease & $85(11.7)$ \\
Injury and poisoning & $83(11.4)$ \\
Coronary artery disease & $71(9.8)$ \\
Pneumonia and pneumonitis & $54(7.4)$ \\
Endocrine and metabolic disease & $34(4.7)$ \\
Other death & $31(4.3)$ \\
Asthma and COPD & $28(3.9)$ \\
Infectious disease & $19(2.6)$ \\
Genitourinary disease & $17(2.3)$ \\
Congestive heart failure & $15(2.1)$ \\
Hypertension & $12(1.7)$ \\
Nervous system disease & $11(1.5)$ \\
Non-atherosclerotic heart disease & $10(1.4)$ \\
Hypotension and vascular disease & $10(1.4)$ \\
Gastrointestinal disease & $10(1.4)$ \\
Mental and behavioral disease & $8(1.1)$ \\
Hepatobiliary and pancreatic disease & $5(0.7)$ \\
Non-traumatic musculoskeletal disease & $4(0.6)$ \\
Shock & $2(0.3)$ \\
Hematologic disease & $1(0.1)$ \\
Missing data & $8(1.1)$ \\
\hline
\end{tabular}

$(\mathrm{aHR}=3.41)$ and lung diseases $(\mathrm{aHR}=2.25)$ such as chronic obstructive pulmonary disease (COPD), asthma, pneumonia, and pneumonitis. Gabayan et al. ${ }^{14)}$ reported a 7-fold risk of 1 -week ED death in patients with noninfectious lung disease such as pleurisy and pneumothorax, 3-fold risk in pneumonia patients, and a 1.7fold risk in patients with COPD patients. Obermeyer et al. ${ }^{13)}$ reported a 3-fold risk of early death in patients discharged from the ED with a diagnosis of dyspnea. Overall, patients with an ED discharge diagnosis of lung disease or dyspnea had an increased risk of early death and our study showed similar trends in older patients. In a previous study, $5.2 \%$ of patients visited ED with dyspnea, and $30 \%$ of whom were discharged. ${ }^{17)}$ Considering the relatively large number of patients with dyspnea discharge, sufficient evaluation and risk stratification is needed.

Gunnarsdottir and Rafnsson $^{12)}$ analyzed 19,259 patients discharged from the ED and found that 63 patients died within 8 days. The causes of death were neoplasm (27\%), CAD (20.6\%), cerebrovascular disease (19\%), and respiratory disease (9.5\%). Rafnsson and Gunnarsdottir ${ }^{11)}$ reported that 156 of 228,097 patients died within 8 days after ED discharge. The causes of death were $\mathrm{CAD}(24.4 \%)$, neoplasm (15.4\%), and cerebrovascular disease $(12.2 \%)$. In the study by Gabayan et al. ${ }^{14)}$ of 357 patients who died within 7 days after ED discharge, the common causes of death were neoplasm (19.6\%), CAD (17.3\%), and non-atherosclerotic

COPD, chronic obstructive pulmonary disease.

Table 5. Subgroup analyses investigating risk factors for 1-week death after emergency department discharge according to emergency department levels

\begin{tabular}{lccc}
\hline & Adjusted HR & p-value* \\
\hline Regional emergency medical center & & $95 \%$ CI $^{*}$ & $<0.001$ \\
Symptom: dyspnea & 4.91 & $2.37-10.17$ & $<0.001$ \\
Neoplasm & 4.63 & $2.82-7.61$ & $<0.001$ \\
Coronary artery disease & 3.43 & $1.66-7.11$ & $<0.001$ \\
Local emergency medical center & & & $<0.001$ \\
Neoplasm & 4.53 & $3.18-6.44$ & $<0.001$ \\
Hypotension and vascular disease & 4.27 & $2.00-9.10$ & $<0.001$ \\
Coronary artery disease & 3.37 & $2.06-5.49$ & 0.032 \\
Symptom: dyspnea & 2.48 & $1.52-4.04$ & $<0.001$ \\
Hepatobiliary and pancreatic disease & 2.29 & $1.07-4.88$ & $<$ \\
Respiratory disease & 1.98 & $1.35-2.92$ & $<0.001$ \\
Local emergency medical facility & & & $<0.001$ \\
Hypotension and vascular disease & 9.17 & $4.03-20.91$ & $<0.001$ \\
Neoplasm & 6.49 & $3.91-10.76$ & $<0.001$ \\
Coronary artery disease & 5.59 & $3.14-9.96$ & $<0.001$ \\
Symptom: dyspnea & 4.79 & $2.97-7.74$ & $<0.001$ \\
Cerebrovascular disease & 3.71 & $2.36-5.83$ & $1.98-4.27$ \\
Respiratory disease & 2.91 & & \\
\hline
\end{tabular}

Discharge diagnoses with significantly increased risks are shown.

$\mathrm{HR}$, hazard ratio; $\mathrm{CI}$, confidence interval.

${ }^{*}$ Cox proportional hazard regression analyses. 
heart disease (11.3\%). These results are similar to our findings that neoplasm, cerebrovascular disease, and $\mathrm{CAD}$ were the main causes of short-term death. Of note is the increasing rate of cerebrovascular disease and CAD-related mortality in Korea. ${ }^{18)}$ In our study, senility was the second leading cause of death, likely because many physicians may indicate unclear diagnoses as the cause of death. ${ }^{19)}$

Subgroup analysis showed increased aHR for hypotension and vascular disease as the ED level increased. Similar to of total included patients, symptoms of dyspnea, as well as neoplasm and $\mathrm{CAD}$ accounted for the highest risks of 1-week ED death in all three groups. The aHRs for these risk factors were highest in local emergency medical facilities compared to those in the other two subgroups. In particular, hypotension and vascular disease had the highest aHR of 9.17.

This study has several strengths. This nationwide population-based study focused on older patients had a large sample size including both men and women. Understanding these patients will be helpful because the number of older patients visiting EDs is increasing. However, this study has some limitations that should be considered when interpreting the results. First, we could not distinguish between patients with hopeless discharge and patients who died unexpectedly. For example, our data did not contain information on do-not-resuscitate orders. Second, the cause of death and discharge diagnoses were recorded by clinicians and could have been inaccurate. However, considering the large number of the study population, the overall pattern of outcomes could be confirmed. Lastly, the database used in this study was sample data, which might have different characteristics from those of the entire older population. However, as the study included 550,000 individuals, the standard error would be minimal.

In conclusion, the rate of death within 1 week among older patients discharged from the ED was $0.5 \%$. Clinicians should consider the increased risk for short-term mortality among older patients with $\mathrm{ED}$ discharge diagnoses of neoplasm, $\mathrm{CAD}$, and respiratory disease. Neoplasm was the leading cause of short-term death in this population.

\section{CONFLICT OF INTEREST DISCLOSURES}

The researchers claim no conflicts of interest.

\section{ACKNOWLEDGEMENTS}

This study utilized the National Health Insurance Service database (No. NHIS-2019-2-095). The interpretations and conclusions reported here do not represent those of the National Health Insurance Service.

\section{SUPPLEMENTARY MATERIALS}

Supplementary materials can be found via http://doi.org/10.4235/ agmr.19.0029.

\section{REFERENCES}

1. Roberts DC, McKay MP, Shaffer A. Increasing rates of emergency department visits for elderly patients in the United States, 1993 to 2003. Ann Emerg Med 2008;51:769-74.

2. Hwang U, Morrison RS. The geriatric emergency department. J Am Geriatr Soc 2007;55:1873-6.

3. Garcia TC, Bernstein AB, Bush MA. Emergency department visitors and visits: who used the emergency room in 2007 ? NCHS Data Brief 2010;(38):1-8.

4. National Emergency Medical Center. E-Gen [Internet]. Seoul: National Emergency Medical Center; c2019 [cited 2019 Sep 15]. Available from: https://www.e-gen.or.kr/egen/main.do.

5. Strange GR, Chen EH, Sanders AB. Use of emergency departments by elderly patients: projections from a multicenter data base. Ann Emerg Med 1992;21:819-24.

6. McCabe JJ, Kennelly SP. Acute care of older patients in the emergency department: strategies to improve patient outcomes. Open Access Emerg Med 2015;7:45-54.

7. Sanders AB. Care of the elderly in emergency departments: conclusions and recommendations. Ann Emerg Med 1992; 21:830-4.

8. Jung YJ, Yoon JL, Kim HS, Lee AY, Kim MY, Cho JJ. Atypical clinical presentation of geriatric syndrome in elderly patients with pneumonia or coronary artery disease. Ann Geriatr Med Res 2018;21:158-63.

9. Hastings SN, Whitson HE, Purser JL, Sloane RJ, Johnson KS. Emergency department discharge diagnosis and adverse health outcomes in older adults. J Am Geriatr Soc 2009;57:1856-61.

10. Hastings SN, Oddone EZ, Fillenbaum G, Sloane RJ, Schmader $\mathrm{KE}$. Frequency and predictors of adverse health outcomes in older Medicare beneficiaries discharged from the emergency department. Med Care 2008;46:771-7.

11. Rafnsson V, Gunnarsdottir OS. All-cause mortality and suicide within 8 days after emergency department discharge. Scand J Public Health 2013;41:832-8.

12. Gunnarsdottir OS, Rafnsson V. Death within 8 days after discharge to home from the emergency department. Eur J Public Health 2008; 18:522-6.

13. Obermeyer Z, Cohn B, Wilson M, Jena AB, Cutler DM. Early death after discharge from emergency departments: analysis of national US insurance claims data. BMJ 2017;356:j239. 
140 Seunggu $\mathrm{Na}$, et al.

14. Gabayan GZ, Derose SF, Asch SM, Yiu S, Lancaster EM, Poon KT, et al. Patterns and predictors of short-term death after emergency department discharge. Ann Emerg Med 2011;58:551558. e2.

15. Rivera DR, Gallicchio L, Brown J, Liu B, Kyriacou DN, Shelburne N. Trends in adult cancer-related emergency department utilization: an analysis of data from the Nationwide Emergency Department Sample. JAMA Oncol 2017;3:e172450.

16. Gabayan GZ, Gould MK, Weiss RE, Chiu VY, Sarkisian CA. A risk score to predict short-term outcomes following emergency department discharge. West J Emerg Med 2018;19:842-8.
17. Kelly AM, Keijzers G, Klim S, Graham CA, Craig S, Kuan WS, et al. An observational study of dyspnea in emergency departments: the Asia, Australia, and New Zealand Dyspnea in Emergency Departments Study (AANZDEM). Acad Emerg Med 2017;24:328-36.

18. Suh I. Cardiovascular mortality in Korea: a country experiencing epidemiologic transition. Acta Cardiol 2001;56:75-81.

19. Park SH, Lee TY. Analysis and improving ways of factors affecting the ill-defined causes of death of the aged in Korea. Health Policy Manag 2011;21:329-48. 